\title{
Electron beam quality control using an amorphous silicon EPID
}

Beck $J A^{1}$, Budgell GJ ${ }^{1}$, Roberts $D A^{2} \&$ Evans $P M^{2}$

$5 \quad{ }^{1}$ North Western Medical Physics

Christie Hospital NHS Foundation Trust

Withington

Manchester

M20 4BX

$10 U K$

${ }^{2}$ Joint Department of Physics

Institute of Cancer Research

Royal Marsden NHS Foundation Trust

15 Downs Road

Sutton

Surrey

SM2 5PT

$U K$

20

Short Title: Electron beam QC with an amSi EPID 


\begin{abstract}
25 An amorphous silicon EPID has been investigated to determine whether it is capable of quality control constancy measurements for linear accelerator electron beams. The EPID grayscale response was found to be extremely linear with dose over a wide dose range and, more specifically, for exposures of 95-100MU. Small discrepancies of up to $0.8 \%$ in linearity were found at $6 \mathrm{MeV}$ ( 8 and $10 \mathrm{MeV}$ showed better agreement).

30 The shape of the beam profile was found to be significantly altered by scatter in air over the approximately $60 \mathrm{~cm}$ gap between the end of the applicator and the EPID. Nevertheless, relative changes in EPID-measured profile flatness and symmetry were linearly related to changes in these parameters at $95 \mathrm{~cm}$ focus to surface distance (FSD) measured using a 2D diode array. Similar results were obtained at $90^{\circ}$ and

$35270^{\circ}$ gantry angles. Six months of daily images were acquired and analysed to determine whether the device is suitable as a constancy checker. EPID output measurements agreed well with daily ion chamber measurements, with a $0.8 \%$ standard deviation in the difference between the two measurement sets. When compared to weekly parallel plate chamber measurements, this figure dropped to

40 0.5\%. A Monte Carlo (MC) model of the EPID was created and demonstrated excellent agreement between MC-calculated profiles in water and the EPID at $95 \mathrm{~cm}$ and $157 \mathrm{~cm}$ FSD. Good agreement was also found with measured EPID profiles, demonstrating that the EPID provides an accurate measurement of electron profiles. The EPID was thus shown to be an effective method for performing electron beam 45 daily constancy checks.
\end{abstract}




\section{Introduction}

The use of electronic portal imaging devices (EPIDs) as quality control (QC) devices

50 for linear accelerator (linac) X-ray beams has been described by numerous authors. They have been used variously to measure beam flatness and symmetry (Kirby \& Williams 1995, Liu et al 2002), X-ray field size (Samant et al 2002, Chang et al 2004, Baker et al 2005, Parent et al 2006a Mohammadi \& Bezak 2007, Clarke \& Budgell 2008), X-ray/light field co-incidence (Curtin-Savard \& Podgorsak 1997, Dunscomb et

55 al 1999), beam output (Dirkx et al 1995, Vieira et al 2006), low dose linearity and beam start-up characteristics (Budgell et al 2005, Vieira et al 2006), matchlines (Holmberg et al 1994, Curtin-Savard \& Podgorsak 1997), fixed wedge factors (Dirkx et al 1995, Budgell et al 2007) and dynamic wedge profiles (Greer \& Barnes 2007). Moreover, they are now commonly used as an IMRT verification method (e.g. Van 60 Esch et al 2004, van Zijtveld et al 2006) which is effectively an indirect check of standard linac beam QC parameters.

We have previously described the use of an amorphous silicon ( $\mathrm{amSi}$ ) EPID as a quick and simple daily monitoring device for linac photon beams (Budgell et al

65 2007), potentially able to replace a daily check device with a single image acquired for each photon energy available on a linac. The usefulness of this system is limited on a dual modality linac if it can only be used for the photon beams. A second measuring device would still be required to measure the electron beam output thus negating the efficiency advantage of the EPID. The purpose of this work was

70 therefore to determine whether an amSi EPID can be used to measure the consistency of electron beam parameters in a manner similar to that used for photon beams.

The use of EPIDS as a method for electron beam QC has not been previously reported. Their potential for the imaging of electron beams has been recognized.

75 Grimm et al (1999), Pouliet et al (2001), Aubin et al (2002, 2003), Jarry \& Verhaegen (2005), Geyer et al (2006). In all these examples the imaging is carried out using the brehmsstrahlung photons generated within the patient. However, in the work described here the electron beam is directly incident upon the EPID. In order to better understand the image formation of an electron beam directly incident upon an amSi EPID a Monte Carlo model of the electron beam and EPID has been constructed and 
used to investigate the generation of an electron image using an EPID (Parent 2006b, Roberts 2008).

\section{Methods}

\section{Electron image acquisition}

An Elekta Precise linear accelerator with an iViewGT amSi EPID (Elekta Oncology Systems, Crawley, UK) mounted at a fixed FSD of approximately $157 \mathrm{~cm}$ was used for the investigation. The EPID was extended to its default position, central to the axis of the beam. A 20x20 cm electron applicator with an open field lead insert was attached to the linear accelerator for all measurements. The full range of available electron energies on the linac was investigated: 6, 8, 10, 12 and $15 \mathrm{MeV}$. Images were acquired using the standard clinical acquisition software. Images displayed by the EPID had a resolution of 1024 x 1024 pixels and were produced via acquiring a series of frames continuously during the exposure. The frames were integrated into a 64 bit buffer and then renormalised to the highest greyscale value to give a 16 bit image. In addition, the image undergoes a series of modifications by the iViewGT software. A continuous dark current (offset) calibration was applied by the panel. All images were subject to an energy-specific flood field correction, thus minimising variations in individual pixel sensitivity. Finally a bad pixel map replaced known inconsistent pixel

100 values with average values obtained from neighbouring pixels. When used to acquire electron images, the system was found to default to use ????? photon flood-field calibration.

\section{EPID sensitivity to changes in dose and beam profile}

105 Beam output measurements were initially studied over a wide monitor unit (MU) range from 50-1000 MU to check whether the EPID response is linear with dose over a wide dynamic range. Subsequently, more detailed measurements were made at 1 MU intervals over a narrower range of 95-105 MU. This was because daily output measurements are made using $100 \mathrm{MU}$ hence this range covers the normal dosimetric range over which the daily dose is expected to vary. Three images were acquired on each occasion and the mean used for analysis. An in-house developed software program written using the IDL (Interactive Data Language, Research Systems Inc.) programming language was used to convert the images to their original greyscale 
values and subsequently analyse the images. A $50 \times 50$ pixel $(12.5 \times 12.5 \mathrm{~mm})$ region

115 of interest based on the image centre was used by the software for determining output. Flatness and symmetry were obtained by extracting GT (in-plane) and AB (crossplane) profiles from the approximate centre of the panel. The $50 \%$ points on the penumbra were determined and the true beam centre defined as the halfway point between the two $50 \%$ points in each direction. New GT and AB profiles were then extracted based on the true centre, consisting of the average of the 15 central profiles (a width of $3.75 \mathrm{~mm}$ ). Flatness and symmetry were then measured from these profiles as defined by the IEC (International Electrotechnical Commission 1989):

125

$$
\text { Symmetry }=\left(D_{\text {leff }} / D_{\text {right }}\right)_{\max } \times 100
$$

$$
\text { Flatness }=\left(D_{\max } / D_{\min }\right) \times 100
$$

Where $\left(D_{\text {lefi }} / D_{\text {right }}\right)_{\max }$ is the maximum ratio of the dose from two symmetric points either side of the beam centre within the flattened area of the beam and $D_{\max }$ and $D_{\min }$ are the maximum and minimum doses in the profile within the flattened area of the beam. The flattened area along the GT and $\mathrm{AB}$ axes is defined as the area corresponding to the $50 \%$ dose points minus $0.1 \times$ field size as defined by the $50 \%$ level. The profiles were averaged over $2 \mathrm{~mm}$ to obtain symmetry and flatness values.

135 The ability of the EPID to detect changes in beam flatness and symmetry was tested by introducing a series of systematic asymmetries into the electron beam profile. This was achieved by systematically varying the $2 \mathrm{R}$ and $2 \mathrm{~T}$ steering currents using the functionality provided in the linac service mode. Beam profiles were initially measured using a 2D Schuster diode array. We have previously verified that the 140 symmetry and flatness values measured using this device are linearly proportional to those measured using an ion chamber in a water phantom. Symmetry and flatness from the Schuster software were recorded together with the corresponding $2 \mathrm{R}$ and $2 \mathrm{~T}$ error value measured using the linear accelerator ion chamber. The experiment was then repeated, using the same steering current changes as used with the Schuster 145 scanner but this time capturing an image using the EPID. Images were acquired from 100MU exposures. The images were the analysed as described above and the flatness and symmetry values expressed relative to the zero error condition; therefore the symmetry and flatness values shown are not a true value as defined by the IEC 
definition (International Electrotechnical Commission 1989). The reason for this is

150 that due to the shape of the EPID electron profiles (figure 1(b)), the absolute flatness values (of 125-130\%) and to a lesser extent the symmetry values are very different from those measured at $\mathrm{D}_{\max }$ in water or using the Schuster scanner. To enable a direct comparison of the changes in measured symmetry/flatness it was therefore necessary to use relative values. The work was also performed at gantry angles of 90 and 270 degrees to investigate the influence of gantry position on the EPID image obtained.

\section{Daily monitoring}

A six-month study was also carried out to monitor electron beam output and symmetry / flatness on a daily basis. Three 100 MU images were acquired each morning as previously described on a clinical machine at the available electron energies $(6,8$ and $10 \mathrm{MeV})$ prior to starting the working day. The EPID output results were then compared with either weekly parallel plate measurements or daily Farmer ionisation chamber measurements that are used at our centre as the daily output check. Typically both measurements were made within an hour of each other. The data was

165 analysed and inconsistencies and anomalies in the results were used to identify any known problems or modifications that were made to the machine over the 6 month period which may possibly explain the variation in the values.

\section{Monte Carlo Model}

170 A $10 \mathrm{MeV}$ electron model for a 20x20 cm applicator was commissioned and modelled using BEAMnrc (Rogers et al 1995) and DOSXYZnrc (Walters et al 2004). Depth dose curves and water profiles were matched with experimental data. The electron parameters for the model were a truncated Gaussian energy spectrum with a mean of $8.8 \mathrm{MeV}, \mathrm{FWHM}$ of $3.7 \mathrm{MeV}$ and min and max cut-offs of $10.2 \mathrm{MeV}$ and12.2MeV

175 respectively. The electron spot was a circle with radius $0.1 \mathrm{~cm}$. A good match was obtained to depth dose curves but only a reasonable match to the beam profiles. To obtain a better match the thickness/shape of the scattering foils would have to be adjusted. However, a perfect fit was not required in order to address the questions to be studied in this work therefore this model was judged to be adequate for this purpose. To simulate EPID images a previously published model of the iViewGT panel was used (Parent et al 2006b, Roberts 2008). An experimentally acquired EPID image of a 20x20 cm field was compared with a Monte Carlo simulation using the 
previously commissioned Monte Carlo model and a model of the iViewGT panel. It must be noted that the experimental image had the flattening/gain image removed and therefore will contain artefacts relating to the non-uniform sensitivities of different regions of the panel.

\section{Results}

Figure 1(a) shows a typical electron image obtained using the EPID; figure 1(b) is a profile taken through the GT (in-plane) axis of the electron image. It is immediately evident that the electron profile is dissimilar to the familiar profiles normally measured at $d_{\max }$ at an FSD of $95 \mathrm{~cm}$; the EPID profile has a very broad penumbra. The same shape is shared by profiles at all electron energies, with the lower energy profiles displaying a wider penumbra and the higher energy profiles a slightly less broad penumbra. Given this large difference between an EPID-measured electron beam and standard electron QC measurements, the question is whether the relative differences measured in the EPID image can be related to changes measured under standard measuring conditions.

EPID sensitivity to changes in dose

Figure 2 demonstrates a highly linear relationship between delivered monitor units and EPID greyscale over the 50 to $1000 \mathrm{MU}$ range at $15 \mathrm{MeV}$. Similar results were obtained at all other electron energies. The experiment when repeated between 95 and $105 \mathrm{MU}$ produced the graphs in figure 3 for $15 \mathrm{MeV}$ and $6 \mathrm{MeV}$ energies

205 respectively. Again a linear result can be seen, but at the lowest clinical energy of 6 $\mathrm{MeV}$ there is a degree of variation from the linear best fit line at 97 and $102 \mathrm{MU}$ of approximately $+0.8 \%$ and $-0.8 \%$ respectively. This variation is random in appearance with lower and higher dose values showing better agreement. This was extremely reproducible with subsequent repeats of the same measurement. This result was

210 further investigated by measuring the electron output at this energy using a Farmer chamber placed at a similar FSD to the panel of $160 \mathrm{~cm}$ measuring at $15 \mathrm{~mm}$ deep in a Perspex block. This was performed to ascertain whether or not this variation was as a result of limitations within the EPID imaging system or was a real variation in linac output. It can be seen from figure $3 b$ that this variation is not present in the ion 215 chamber results suggesting that the anomalous results at 97 and $102 \mathrm{MU}$ are due to 
the EPID rather than variation in linac output. \{this is very strange. Is it worth comment how many times these experimental points were repeated?\}

\section{Daily output monitoring}

220 The results of 6 months measurements of linac output can be seen in figure 4 for 10 $\mathrm{MeV}$; figure 4(a) includes both weekly parallel plate and daily Farmer chamber readings, figure 4(b) only the parallel plate chamber results. The EPID results were normalised relative to a single parallel plate chamber measurement. This normalisation was reset following any significant hardware changes: for example,

225 EPID panel replacement or recalibration of the EPID. Such modifications of the normalisation occurred on days 43 and 100. Figure 4 displays large fluctuations in machine output in the early days of the study reflected in both the chamber and EPID results. This was a real variation caused by a failing magnetron and hence shows that the EPID is capable of detecting machine output problems.

The study shows a close correlation between the EPID and daily output results (figure 4a) with a standard deviation in the differences of $0.78 \%$ at $10 \mathrm{MeV}$. Inconsistencies in the results can be seen between the EPID panel and the Farmer ionization chamber, particularly between days 20 and 40 but this was resolved by the renormalization of

235 the EPID output on day 43 and over the 6 month period these anomalies are relatively infrequent. When only the more accurate parallel plate chamber results are compared with the EPID results (figure $4 \mathrm{~b}$ ) this figure drops to $0.49 \%$ which suggests that EPID-measured output is an excellent method of detecting relative dose changes in an electron beam. Very similar results were obtained for the other electron energies; at

$24010 \mathrm{MeV}$ the standard deviations were $0.87 \%$ and $0.47 \%$ for all output results / parallel plate chamber only respectively and at $6 \mathrm{MeV}$ the standard deviations were $0.76 \%$ and $0.46 \%$.

On day 38, a striping artefact is known to have developed on the EPID electron 245 images, which lasted until day 100. Although this is the reason why the results from day 43 required renormalization, there was still reasonable agreement between the EPID and chamber results through this period. This suggests that the method is robust even in the presence of image artefacts. The imaging panel was replaced on day 100 and the results renormalized again from that day. 


\section{EPID sensitivity to changes in beam profile}

Figure 5(a) demonstrates the relationship between the induced $2 \mathrm{R}$ error and the recorded percentage GT (in-plane) flatness and symmetry values from both the Schuster scanner and EPID panel for $6 \mathrm{MeV}$. A direct linear relationship can be seen

255 between the two for both Schuster and EPID results; this makes the panel suitable for detecting changes in beam symmetry and flatness. Figure 5(b) shows the relationship between the induced $2 \mathrm{~T}$ error and the recorded percentage $\mathrm{AB}$ (cross-plane) flatness and symmetry value from the Schuster scanner and EPID panel for $15 \mathrm{MeV}$. Due to the difficulty in producing tilts in the $\mathrm{AB}$ axis above approximately $4 \%$, figure $5(\mathrm{~b})$ is plotted with the scale suitably adjusted. Again, a near-linear relationship can be seen between the $2 \mathrm{~T}$ error value and the flatness and symmetry figures recorded for both devices. Similar results were observed for the other energies measured in all cases. The results obtained at $90^{\circ}$ and $270^{\circ}$ gantry angle for multiple electron energies were also very similar to those of figures $5(\mathrm{a}) \&(\mathrm{~b})$.

The 6 month study results for EPID symmetry and flatness can be seen in figure 6 for $8 \mathrm{MeV}$. EPID results were measured and then subtracted from the initial result obtained on day hence the results indicate percentage change in symmetry and flatness relative to this date. Symmetry and flatness results were collected until day

270 38. The electron image artefacts present between days 38 and 100 prevented symmetry and flatness values from being measured hence these results have been omitted and results recorded again from day 101 when the panel had been replaced. Days 39-123 in figure 6 therefore correspond to days 101-185 in figure 4.

275 Detailed analysis of the results shows that the $\mathrm{AB}$ symmetry and flatness are extremely stable, as expected since the $\mathrm{AB}$ plane is unaffected by beam bending and variations in beam energy. The GT values are much more variable; however they do appear to be more variable after the installation of the second panel. The reason for this is not known - there is nothing in the QC results from this period to suggest that

280 this is a real linac effect so it appears to be connected with the EPID. But the levels of variation are still relatively low so this is not a cause for concern.

\section{Monte Carlo modelling}


Figure 7a shows a Monte Carlo calculated profile for the EPID overlaid onto the 285 experimentally measured EPID profile. Good agreement is seen between the two profiles. To investigate whether measuring with the EPID significantly influences the electron profile, EPID profiles were compared to water tank profiles at the normal detector distance $(\mathrm{SSD}=157.1 \mathrm{~cm})$ and at the normal experimental distance $(\mathrm{SSD}=95 \mathrm{~cm})$. To take into account the different densities of the various EPID layers,

290 profiles in water were obtained at $1.76 \mathrm{~cm}$ deep; the water equivalent depth for the iViewGT panel. This number was obtained by multiplying each EPID layer's density by its thickness and adding them together. The total gives the equivalent thickness of water. As the image is formed in the gadox scintillator layer the above procedure was only conducted for layers up to the amorphous silicon diodes i.e. the last layer was gadox. Figure 7(a) shows that the Monte Carlo EPID profiles, experimental EPID profiles and equivalent depth Monte Carlo water profiles are in good agreement at $157 \mathrm{~cm}$ FSD whilst figure 7(b) shows good agreement between Monte Carlo EPID profiles and equivalent depth Monte Carlo water profiles at 95cm FSD. This therefore highlights the EPID profiles are mainly affected by the electrons scattering in air.

\section{Discussion}

The first observation from the results reported in this paper is that the EPID studied was found to be a suitable method for measuring both electron output consistency and beam profile consistency. This was by no means a foregone conclusion. EPIDs are

305 specifically designed for imaging photons and, unlike photon beams, electron beams undergo significant degradation due to scattering interactions with electrons in the air gap between the end of the applicator and the EPID. However, the experimental results demonstrate that despite this high level of scatter, the EPID-measured output is highly linear with dose and that changes in the beam profile measured with the EPID

310 are linearly proportional to changes measured at $\mathrm{D}_{\max }$. The Monte Carlo simulation of the EPID demonstrated that the EPID is capable of imaging electrons accurately, with the EPID profiles shown to be the same as water profiles at both 95 and $157 \mathrm{~cm}$ FSD. When photons are incident on this type of EPID they are first converted to electrons and then to light photons for detection by the phototransistors in the amorphous 315 silicon - the so-called indirect detection method. It is not surprising therefore that an electron beam is capable of being imaged. What was not certain was whether the 
thickness of the layers in the EPID, designed to ensure sufficient photons interact to produce a high enough signal, would not be too thick to yield a reasonable signal from an electron beam. This work demonstrates that this is not the case. The previously reported examples of electron images through a patient were generated not directly by electrons but by the brehmstrahhlung photons generated within the patient hence direct comparison with these results is not meaningful.

These results are particularly encouraging in that they confirm that our previously reported technique for daily monitoring of photon beams can be readily extended to electron beams as well, making the EPID a suitable device to replace daily output check devices. The results reported here were based on the mean of three images per day, as it was felt that the electron measurements might be more variable than photon measurements. However, the reproducibility of the results on any one day was found to be excellent - hence only one image per energy per day would be required, as for photons. The results also suggest that the EPID will be suitable for post-service checks and checking beam profile variation with gantry angle, as previously suggested for photons (Budgell et al 2007).

335 The anomalies seen in the dose linearity at $6 \mathrm{MeV}$ suggest that using the EPID for an output check device might be less accurate at this low energy. A discrepancy of $0.8 \%$, whilst readily measurable, is still acceptable if using the device as a constancy checker for which a tolerance of 5\% would typically be applied. Moreover, the actual result from 6 months comparison with daily ion chamber results showed that the $6 \mathrm{MeV}$ results were no less accurate than the 8 or $10 \mathrm{MeV}$ results, thus suggesting that this effect is not a significant problem in practice. This may be because on a daily basis it is unusual for the machine output to be close to the values where the discrepancies were observed.

345 The EPID is plainly only suitable as a consistency checker. If discrepancies are found by the EPID measurements will still need to be made more accurately at $95 \mathrm{~cm}$ FSD since the beam shape is so different at $157 \mathrm{~cm}$ FSD. There were also changes found with EPID calibration and when an artefact developed in the electron beam images. Interestingly, the same artefact was not observed in the photon images acquired during the same period. Another effect causing a difference between the actual 
incident profile and the measured profile is the fact that the EPID automatically applies a flood field derived from a photon field. This will introduce a systematic error since the electron profiles will be divided by the photon beam profile inherent in the flood field. However, since the same flood field is used each time, this is a constant systematic error and does not affect the use of the EPID as a consistency check device.

We have recently reported the development of software interfaced to an EPID of this type to enable daily monitoring using an EPID to be performed automatically without user intervention whenever a specified beam is run (Mackay et al 2007). This software is applicable both to photons and to the electron measurements described in this paper. This automation completes the chain to allow rapid daily output checks for electrons and photons using an amSi EPID in only the time taken to deploy the EPID and deliver a beam at each available clinical energy. This method is now undergoing testing in our department.

\section{Conclusions}

An amSi EPID has been demonstrated to be an effective method for measuring electron beam output and beam profile parameters and is thus suitable as a device for rapid linac daily constancy checks.

\section{Acknowledgements}

DAR is partially funded by Elekta limited

\section{References}

Aubin M, Faddegon B and Pouliot J, Electron Beam Verification with an a-Si Flat Panel Electronic Portal Imaging Device. SPIE - The Int. Society for Optical Eng., Medical Imaging, San Diego, Feb. 2002.

Aubin M, Langen K, Faddegon B and Pouliot J, Electron Beam Verification with an a-Si EPID, Annual Meeting of AAPM, San Diego, August. 2003.

Aubin M, Faddegon B and Pouliot J Clinical Implementation of Electron Beam Verification with an a-Si EPID, Annual Meeting of AAPM, Montréal, July 2002. 
Aubin M, Faddegon B and Pouliot J, Electron Beam Verification with Electronic Portal Imaging Devices, SPIE-The Int. Society for Optical Eng., Medical Imaging, 385 San Diego, Feb. 2002.

Pouliot J, Aubin M and Faddegon B 2001 Electron MultiLeaf Collimator Beam Verification with Electronic Portal Imaging Devices, Int. J. Radiation Oncology Biol. Phys. 51 (3) (supp)), 313. 2001. Meeting: 43th ASTRO Annual Meeting, San Francisco.

390 Baker S J K, Budgell G J and Mackay R I 2005 Use of an amorphous silicon electronic portal imaging device for multileaf collimator quality control and calibration Phys. Med. Biol. 50 1377-1392.

Budgell GJ, Zhang Q, Trouncer RJ, Mackay RI. 2005 Improving IMRT quality control efficiency using an amorphous silicon electronic portal imager. Med Phys.

$395 \quad 32(11) 3267-3278$.

Budgell GJ, Zhang R, Mackay RI 2007 Daily monitoring of linear accelerator beam parameters using an amorphous silicon EPID. Phys Med Biol. 52(6) 1721-33.

Chang J, Obcemea CH, Sillanpaa J, Mechalakos J, Burman C. 2004 Use of EPID for leaf position accuracy QA of dynamic multi-leaf collimator (DMLC) treatment, Med. 400 Phys. 31, 2091-2096.

Clarke MF and Budgell GJ 2008 Use of an amorphous silicon EPID for measuring MLC calibration at varying gantry angle. 2008 Phys Med Biol. 53(2):473-85.

Curtin-Savard A and Podgorsak E B 1997 An electronic portal imaging device as a physics tool, Med. Dosim. 22, 101-105.

405 Dirkx M L, Kroonwijk M, de Boer J C and Heijmen B J 1995 Daily dosimetric quality control of the MM50 Racetrack Microtron using an electronic portal imaging device. Radiother. Oncol. 37, 55-60.

Dunscombe P, Humphreys S and Leszczynski K 1999 A test tool for the visual verification of light and radiation fields using film or an electronic portal imaging 410 device. Med. Phys. 26, 239-243.

Geyer P, Baus WW, Baumann M. 2006 Portal verification of high-energy electron beams using their photon contamination by film-cassette systems. Strahlenther Onkol. 182 37-44. 
Greer PB and Barnes MP. 2007 Investigation of an amorphous silicon EPID for

415 measurement and quality assurance of enhanced dynamic wedge. Phys Med Biol. 52(4) 1075-87.

Grimm DF, Gillin MT, Kline RW, Janjan N. 1989 Electron beam port films. Med Dosim. 14(1) 31-33.

Holmberg O, Huizenga H, Idzes MH, Lebesque JV, Vijlbrief RE, Mijnheer BJ. 1994

420 In vivo determination of the accuracy of field matching in breast cancer irradiation using an electronic portal imaging device. Radiother Oncol. 33(2) 157-66.

Jarry G, Verhaegen F. 2005 Electron beam treatment verification using measured and Monte Carlo predicted portal images. Phys Med Biol. 50(21) 4977-94.

Kirby MC, Williams PC. 1995 The use of an electronic portal imaging device for exit 425 dosimetry and quality control measurements. Int J Radiat Oncol Biol Phys. 31(3) 593-603.

Liu G, van Doorn T, Bezak E. 2002 Assessment of flatness and symmetry of megavoltage $\mathrm{x}$-ray beam with an electronic portal imaging device (EPID). Australas Phys Eng Sci Med. 25(2) 58-66.

430 Mackay RI, Smith S \& Budgell GJ. EPIQC: a software tool for fully automatic daily quality control of linear accelerators using amorphous silicon EPIDs. Page 88 of the Proceedings of the XVth International Conference on the Use of Computers in Radiation Therapy, Toronto, Canada, June 2007 Mohammadi M and Bezak E 2007 Evaluation of MLC leaf positioning using a 435 scanning liquid ionization chamber EPID. Phys Med Biol. 52 21-33.

Parent L, Seco J, Evans PM, Dance DR and Fielding A. 2006 Evaluation of two methods of predicting MLC leaf positions using EPID measurements. Med. Phys. 33 3174-3182.

Parent L, Seco J, Evans P M, Fielding A and Dance D R 2006 Monte Carlo modelling 440 of a-Si EPID response: The effect of spectral variations with field size and position Medical Physics 33 4527-4540

Roberts DA, Hansen VN, Niven AC, Thompson MG, Seco J and Evans PM 2008 A low $\mathrm{Z}$ linac and flat panel imager: Comparison with the conventional imaging approach Phys. Med. Biol. (in press). 
Rogers D W O, Faddegon B A, Ding G X, Ma C M, We J and Mackie T R 1995 Beam - A Monte-Carlo Code to Simulate Radiotherapy Treatment Units Medical Physics 22 503-524

Samant S S, Zheng W, Parra N A, Chandler J, Gopal A, Wu J, Jain J, Zhu Y and

450 Sontag M. 2002 Verification of multileaf collimator leaf positions using an electronic portal imaging device Med. Phys. 29 2900-2912.

Van Esch A, Depuydt T, Huyskens DP. 2004 The use of an aSi-based EPID for routine absolute dosimetric pre-treatment verification of dynamic IMRT fields.

Radiother Oncol. 71(2) 223-34.

455 van Zijtveld M, Dirkx ML, de Boer HC, Heijmen BJ. 2006 Dosimetric pre-treatment verification of IMRT using an EPID; clinical experience. Radiother Oncol. 81(2) 168-175.

Vieira S C, Bolt R A, Dirkx M L, Visser AG and Heijmen BJ. 2006 Fast, daily linac verification for segmented IMRT using electronic portal imaging. Radiother Oncol.

$460 \quad 8086-92$.

Vieira S C, Dirkx M L, Pasma K L and Heijmen B J 2002 Fast and accurate leaf verification for dynamic multileaf collimation using an electronic portal imaging device. Med. Phys. 29 2034-2040.

Walters B, Kawarakow I, \& Rogers D W O 2004, DOSXYZnrc users manual NRCC 465 Report PIRS-794revB NRCC, Ottawa, Canada.

Yang Y and Xing L 2004 Quantitative measurement of MLC leaf displacements using an electronic portal image device Phys. Med. Biol. 49 1521-1533. 
(a)

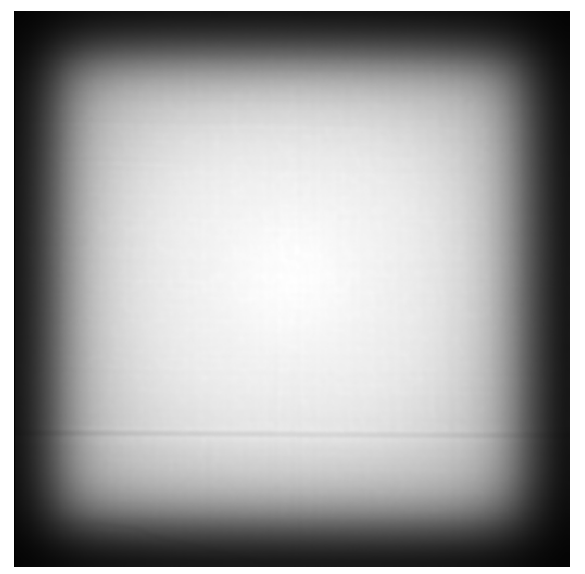

(b)

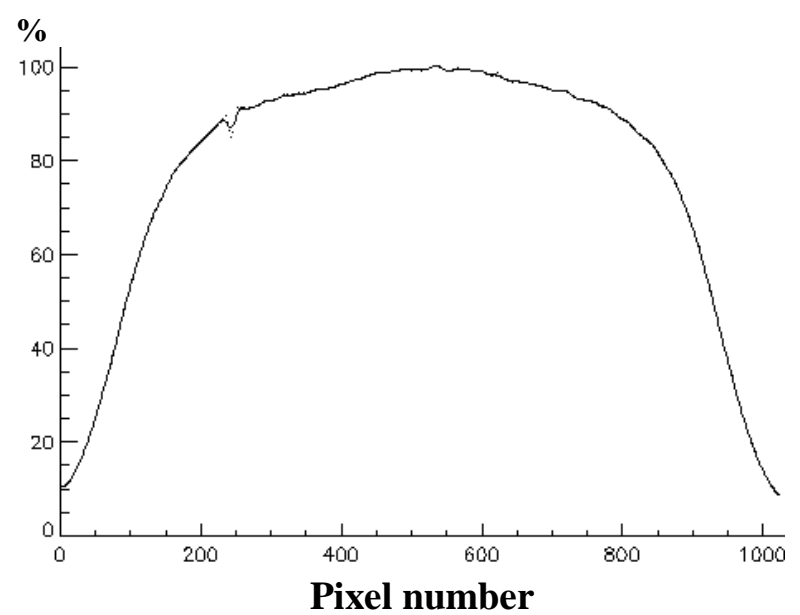

Figure 1 (a) A $10 \mathrm{MeV}$ EPID image of a 20x20 cm electron field. (b) An in-plane (GT) profile through the image. Higher and lower energies display the same shape but sharper/broader penumbrae respectively. 


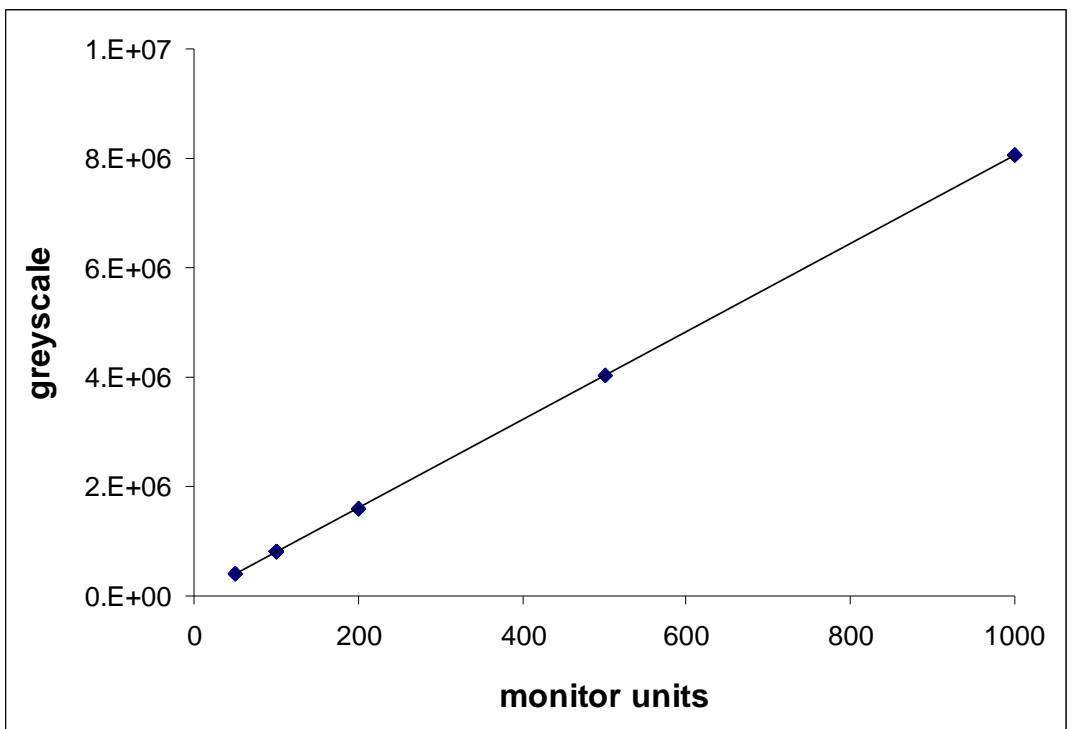

Figure 2 Relationship between delivered monitor units and greyscale at $15 \mathrm{MeV}$. 
(a)

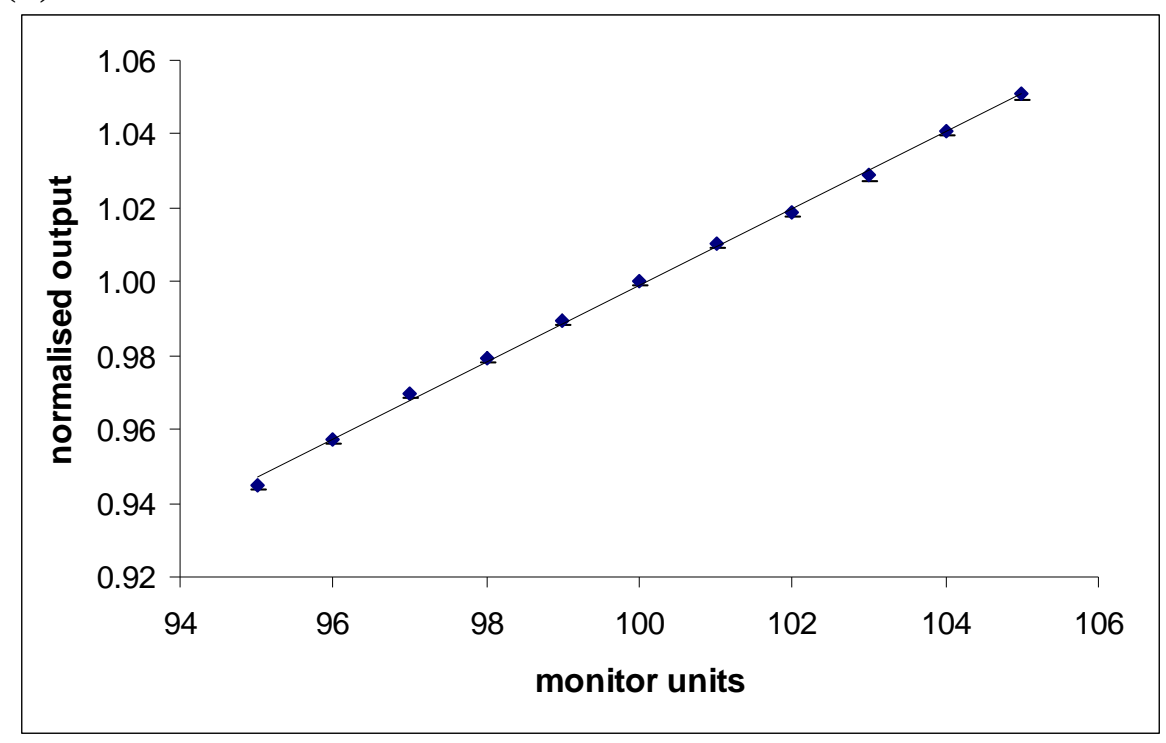

(b)

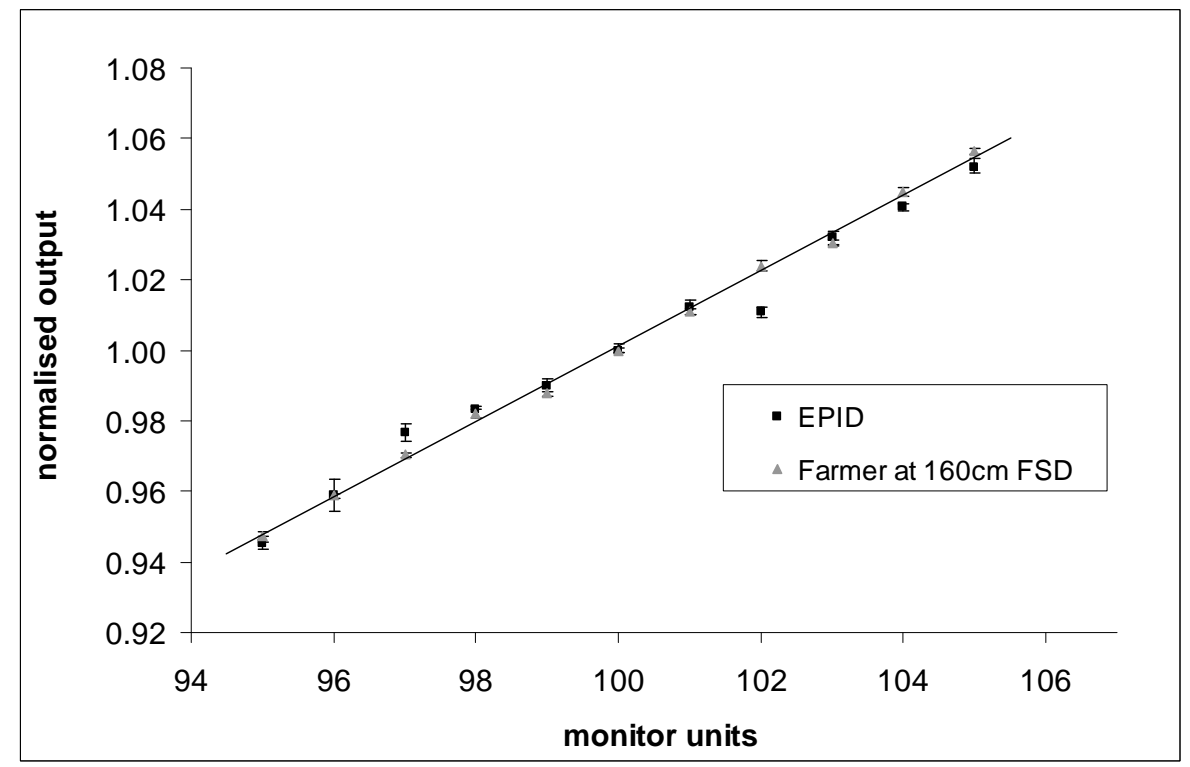

Figure 3 Relationship between delivered monitor units and normalized greyscale at (a) $15 \mathrm{MeV}$ and (b) $6 \mathrm{MeV}$ for 95-105 MU. (b) also shows the normalized dose vs mu results for a Farmer chamber measurement at the same FSD as the EPID panel. 
(a)

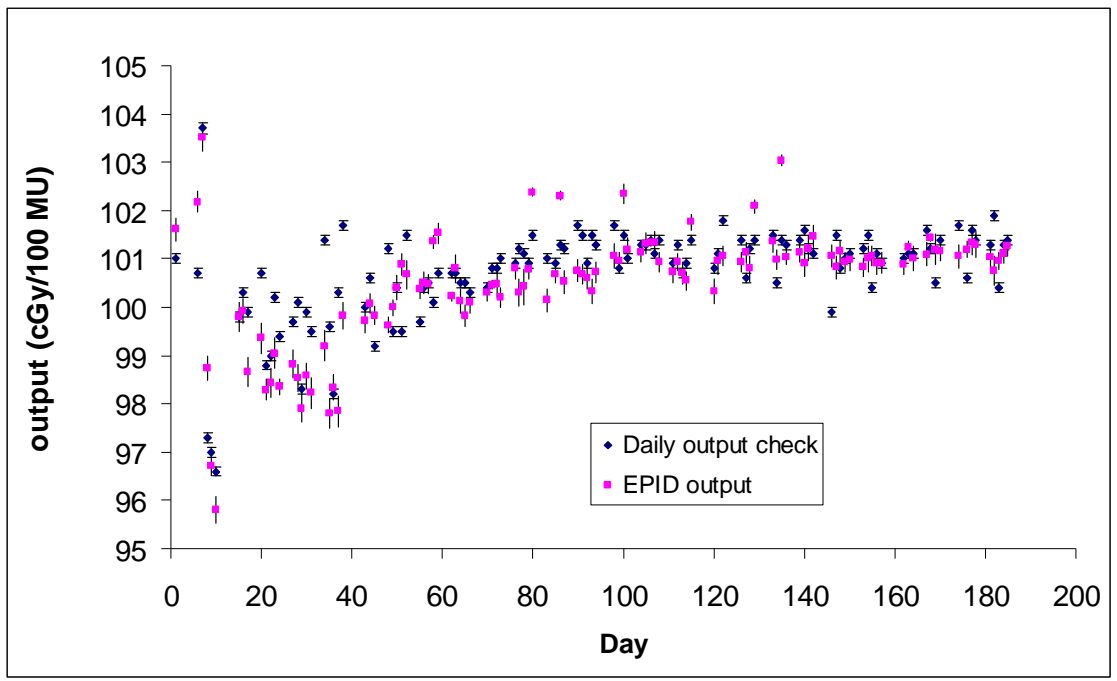

(b)

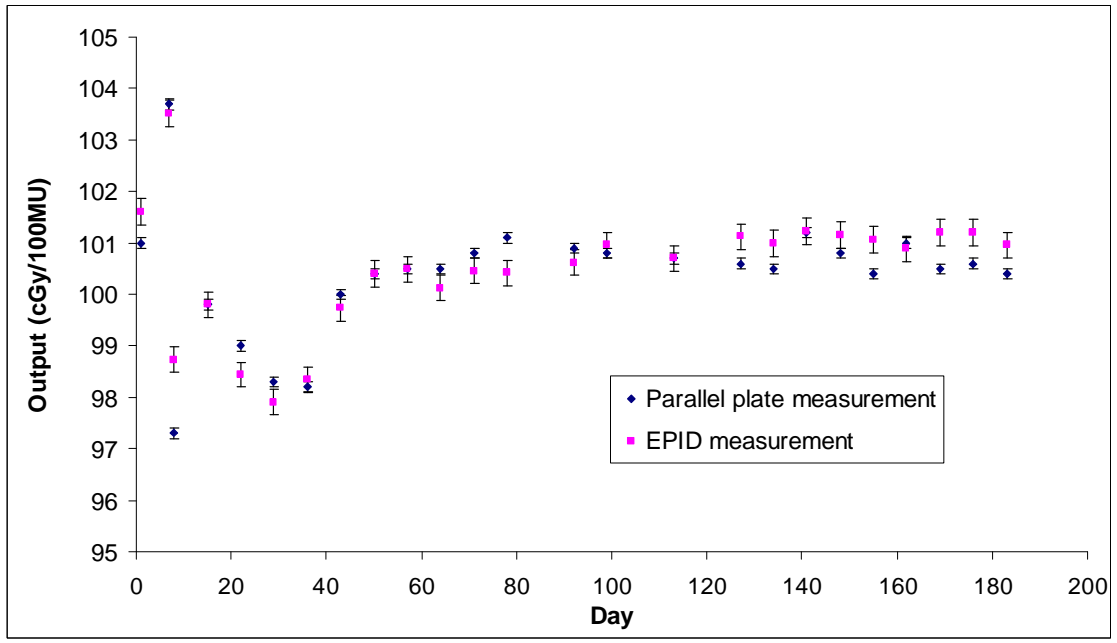

Figure 4 Six month study results at $10 \mathrm{MeV}$ of (a) EPID and ion chamber daily output check. (b) EPID and weekly parallel plate absolute dose measurement. 
(a)

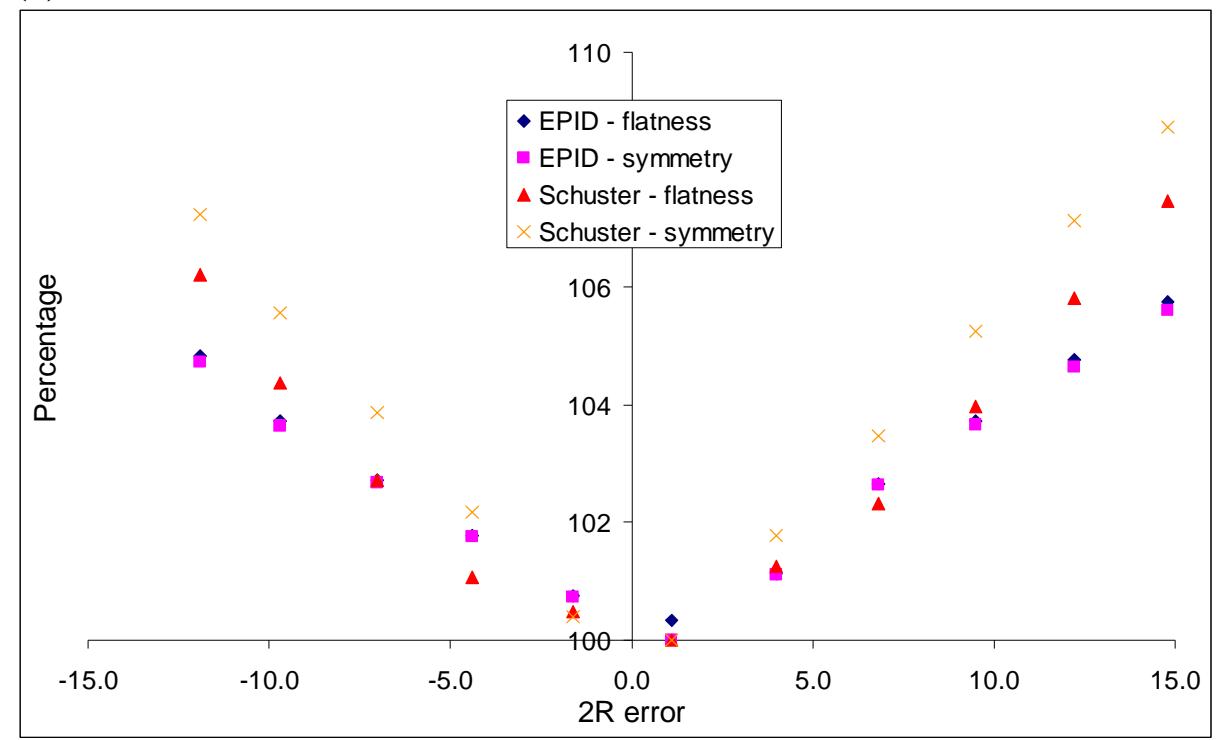

(b)

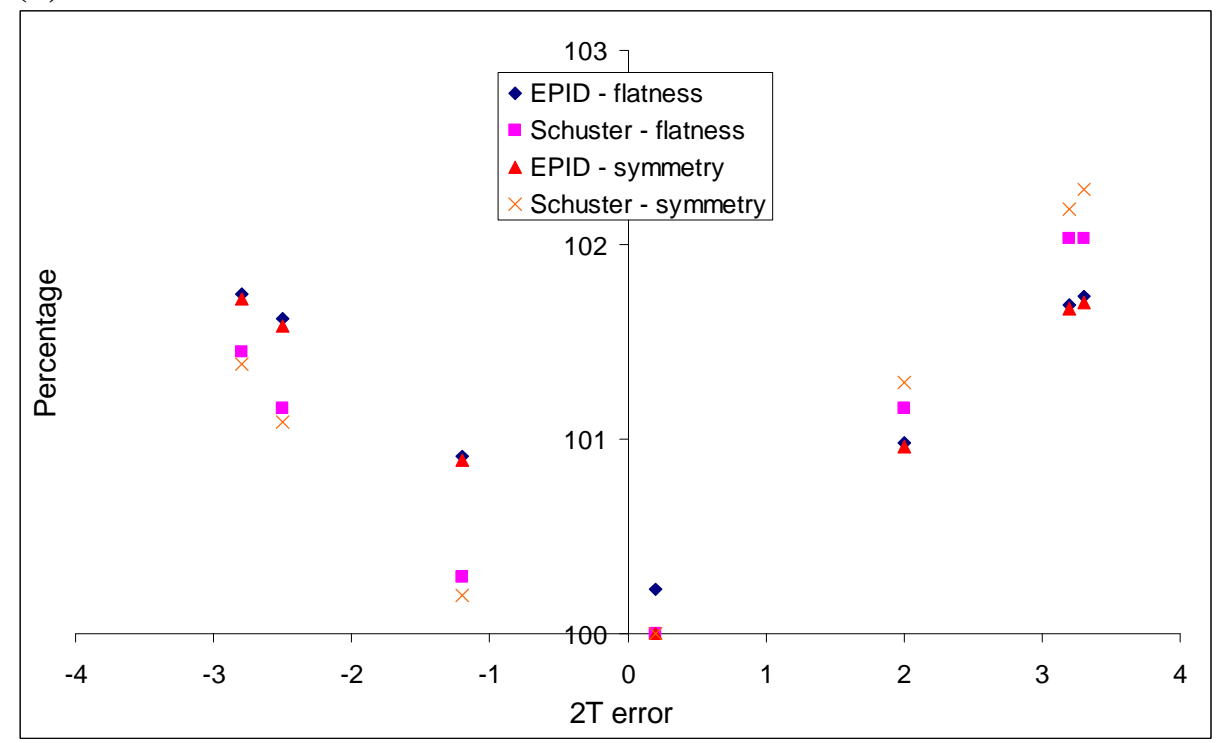

Figure 5 EPID and Schuster symmetry and flatness values normalized to 100 at zero error versus linac error signal. For (a) $6 \mathrm{MeV} 2 \mathrm{R}$ profiles, (b) $15 \mathrm{MeV} 2 \mathrm{~T}$ profiles. Note the axes scales are much smaller in (b) since the beam could not be made any more asymmetric in this plane. 


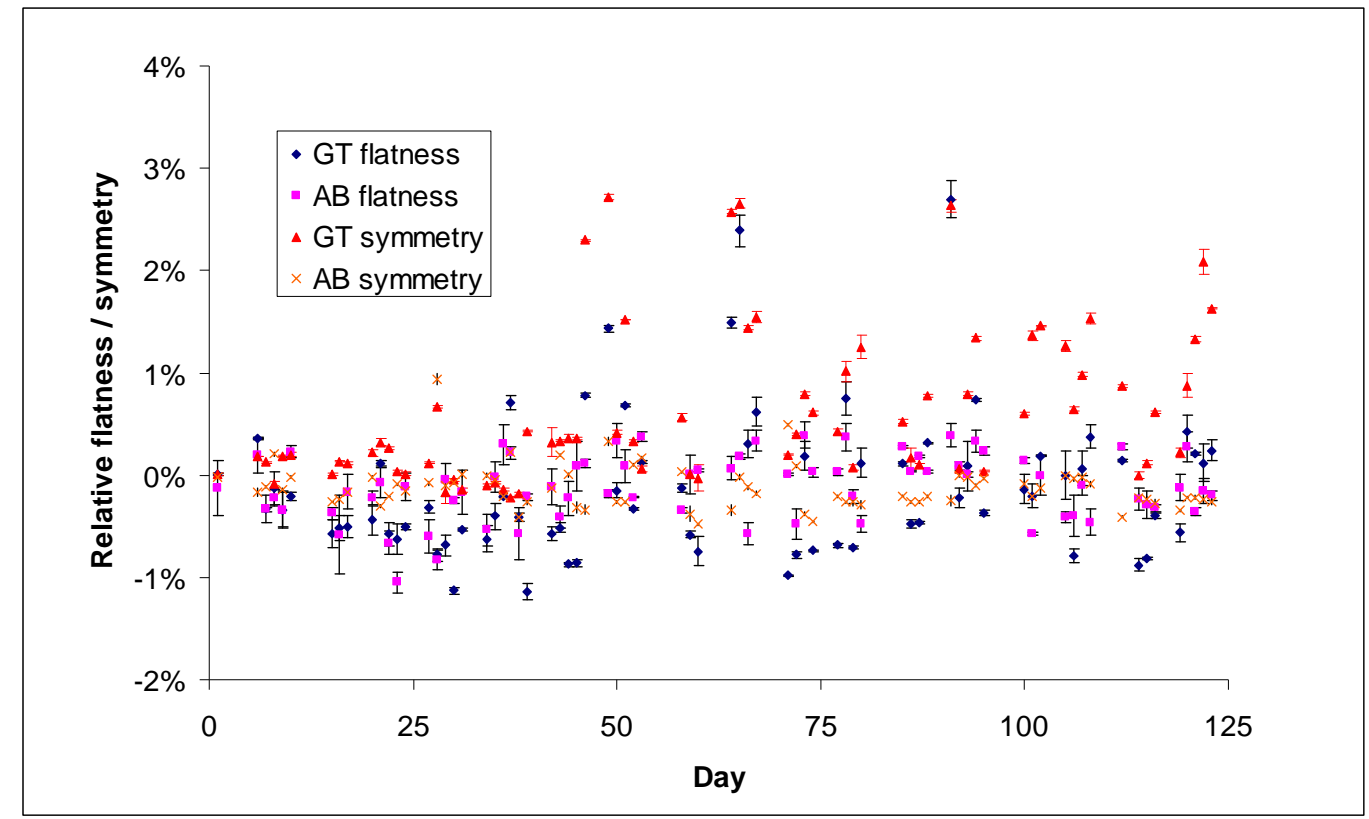

Figure $68 \mathrm{MeV}$ EPID measured symmetry and flatness results over a 6 month period - a period during which an artefact existed and sensible results were unobtainable has been omitted. 


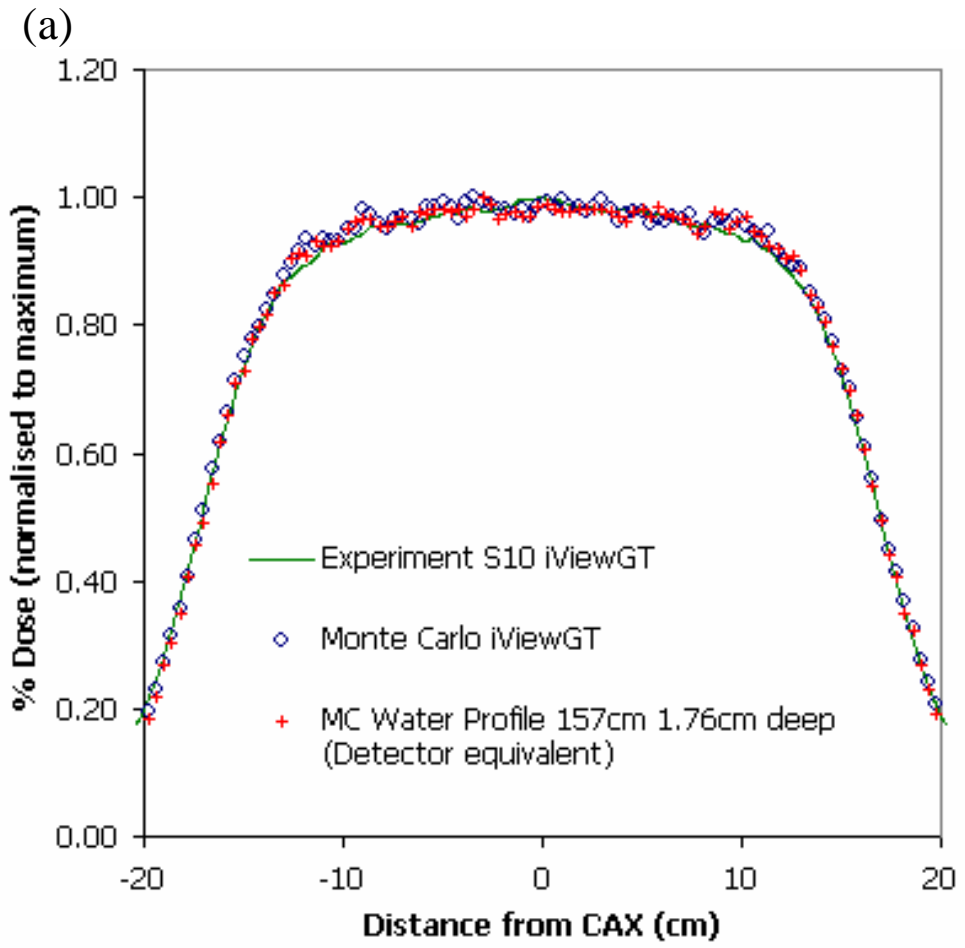

(b)

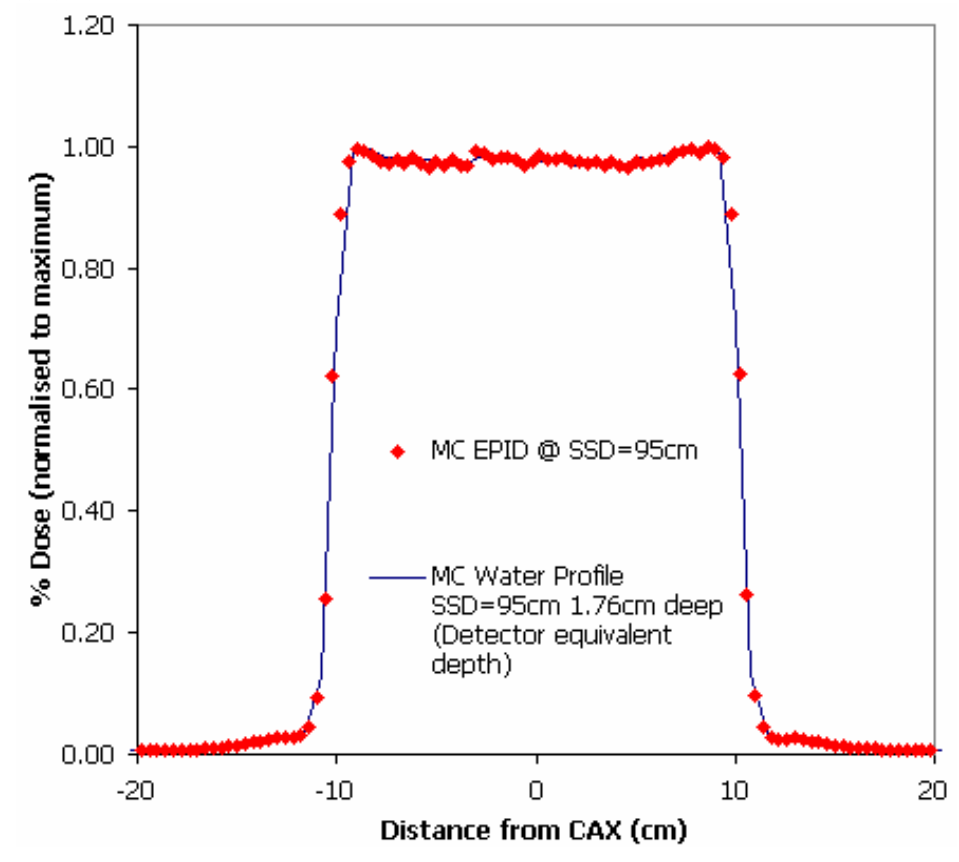

Figure 7 (a) Monte Carlo calculated EPID and water profiles at SSD $=157.1 \mathrm{~cm}$ overlaid onto the EPID-measured profile. (b) Monte Carlo calculated EPID and water tank profiles at $\mathrm{SSD}=95 \mathrm{~cm}$ \{can you use the same symbols in the 2 graphs? 